\title{
Aplicación de un Plan de Cuidados Enfermeros utilizando el modelo de Virginia Henderson (catorce necesidades) a una persona que presenta lesiones causadas por pénfigo vulgar
}

\author{
Laura Navarrete Cortés, * Sara Santiago García **
}

\begin{abstract}
RESUMEN
Presentamos un caso clínico cuyo interés es mostrar el Plan de Atención de Cuidados Enfermeros (PLACE). La metodología guiada está basada en la taxonomía NANDA, NOC y NIC, la cual utiliza el modelo de Virginia Henderson de las catorce necesidades, esto con la finalidad de difundir la labor diaria del profesional de enfermería. En el PLACE planteamos la posibilidad también, de referirse a éste, como tratamiento enfermero en un futuro más próximo, cuyos objetivos son aplicar los conocimientos científicos fundamentados, razonados y planeados, a fin de resolver necesidades de salud centrados en la persona desde su padecimiento hasta su rehabilitación, orientados con su método principal: el Proceso de Atención de Enfermería (PAE), el cual permite aplicar cuidados a personas sanas o enfermas, pudiéndose aplicar desde una clínica de heridas y ostomías hasta para la gran diversidad de personas en distintas edades que se encuentren en servicios de hospitalización, clínica o a domicilio, así como en aquellas que presenten diferentes necesidades de atención en su salud. El caso clínico difundido es de una mujer con diagnóstico médico de pénfigo vulgar, que presenta lesiones epidérmicas en una gran superficie corporal y úlceras por presión de IV grado a nivel sacro, la cual es intervenida interdisciplinariamente en una Clínica de Heridas y Ostomías (CHO). De acuerdo con lo antes mencionado, haremos énfasis en el tratamiento de enfermería.
\end{abstract}

Palabras clave: Cuidado enfermero-tratamiento enfermero, plan de cuidados enfermero, modelo teórico.

\section{Implementation of a plan of care using the model of Virginia Henderson (fourteen needs), to a person who presents lesions caused by pemphigus vulgaris}

\begin{abstract}
We present a clinical case where the interest is to show a Plan of Nursing Care (PLACE) with a guided methodology based on the taxonomy NANDA, NIC and NOC using the Virginia Henderson model of the fourteen needs, with the purpose of disseminating the daily work of the nursing professional; for the purpose of disseminating the daily work of the nursing professional; in the place we have the possibility also to refer to this as treatment nurse in a near future; whose objectives are to apply sound scientific knowledge, reasoned and planned, in order to resolve health needs focusing on the person from their disease, and follow him to his rehabilitation, oriented with its main method: The process of Nursing Care (PAE), that allows you to apply care to people healthy or diseased, which could be applied from a clinic of wounds and ostomies, to the
\end{abstract}

* Licenciada en Enfermería, Unidad de Especialidades Centro Médico Nacional Siglo XXI Instituto Mexicano del Seguro Social. ** Maestra en Enfermería. Instituto Nacional de Neurología y Neurocirugía "Manuel Velasco Suárez".

Correspondencia: Lic. Laura Navarrete Cortés

E-mail:_aura286_1@hotmail.com; santiago90_03@yahoo.com.mx

Este artículo puede ser consultado en versión completa en http://www.medigraphic.com/enfermerianeurologica 
great diversity people in different ages, who are in hospitalization services, clinic or home, as well as in those who present different care needs in their health. He clinical case spread, is a female person with a medical diagnosis of pemphigus vulgaris, which presents epidermal injury in a large body surface area and pressure ulcers of level IV degree to sacrum, which is operated in an interdisciplinary way in a clinic of wounds and Ostomies (CHO). According to the described we will place emphasis on the treatment of nursing.

Key words: Care nurse-treatment nurse, plan of nursing care, theoretical model.

\section{JUSTIFICACIÓN}

Actualmente existe una gran variedad de información acerca de cómo realizar un Proceso de Atención de Enfermería o realizar un Plan de Atención de Cuidados Enfermeros (PLACES), sin embargo, aún quedan desfasadas las intervenciones de enfermería con la utilización de dichas metodologías, aunado a los vacíos dentro de la evidencia cotidiana en la aplicación de ambos planes. El presente trabajo lo realizamos con el objetivo principal de compartir evidencia de nuestra labor en la profesión.

\section{OBJETIVO}

El presente trabajo tiene por objetivo configurar un PLACE individualizado donde se demuestre una guía metodológica acerca de las intervenciones de enfermería, esto con la finalidad de aumentar la calidad y garantía del cuidado enfermero a una persona que se encuentra padeciendo lesiones dérmicas de pénfigo vulgar.

\section{MARCO TEÓRICO}

La Organización Mundial de la Salud (OMS) en 1977 definió al Proceso de Atención de Enfermería (PAE) como "un sistema de intervenciones propias de Enfermería sobre la salud de los individuos, las familias, las comunidades, o ambos". Para la Mtra. Alfaro-Lefevre, la forma dinámica y sistematizada de brindar cuidados enfermeros consta de cinco etapas: valoración, diagnóstico, planificación, ejecución y evaluación. ${ }^{2}$ ¿Para qué se aplica? Este proceso proporciona las bases para habilidades de pensamiento crítico, con un razonamiento clínico requerido para actuar en forma segura y efectiva, además con juicio clínico., ${ }^{2,3}$

Mientras que para Griffith-Kenney y Christensen el Plan de Cuidados Enfermeros (PLACE) "es un instrumento para documentar y comunicar la situación del paciente-cliente, los resultados que se esperan, las estrategias, indicaciones, intervenciones y la evaluación de todo ello".4

En los lineamientos generales para la elaboración del PLA$\mathrm{CE}$, se mencionan los diferentes tipos. A saber: ${ }^{5}$

Cuadro I.

\begin{tabular}{|c|c|}
\hline Necesidad alterada & Diagnóstico enfermero \\
\hline 1. Respirar normalmente. & No aplica \\
\hline 2. Comer y beber adecuadamente. & No aplica \\
\hline 3. Eliminar por todas las vías corporales. & No aplica \\
\hline 4. Moverse y mantener posturas adecuadas. & Diagnóstico deterioro de la movilidad física \\
\hline 5. Dormir y descansar. & No aplica \\
\hline 6. Escoger la ropa adecuada. Vestirse y desvestirse. & No aplica \\
\hline $\begin{array}{l}\text { 7. Mantener la temperatura corporal dentro de los límites normales adecuando } \\
\text { la ropa y modificando el ambiente. }\end{array}$ & No aplica \\
\hline 8. Mantener la higiene corporal y la integridad de la piel. & $\begin{array}{l}\text { Diagnóstico deterioro de la integridad cutánea } \\
\text { Diagnóstico deterioro de la integridad tisular }\end{array}$ \\
\hline 9. Evitar los peligros ambientales y evitar lesionar a otras personas. & $\begin{array}{l}\text { Diagnóstico riesgo de infección. } \\
\text { Diagnóstico dolor agudo }\end{array}$ \\
\hline $\begin{array}{l}\text { 10. Comunicarse con los demás expresando emociones, necesidades, temores } \\
\text { u opiniones. }\end{array}$ & Diagnóstico disposición para mejorar el rol parenteral \\
\hline 11. Vivir de acuerdo con sus propios valores y creencias. & No aplica \\
\hline 12. Ocuparse en algo de tal forma que su labor tenga un sentido de realización personal. & No aplica \\
\hline 13. Participar en actividades recreativas. & No aplica \\
\hline $\begin{array}{l}\text { 14. Aprender, descubrir o satisfacer la curiosidad que conduce a un desarrollo normal } \\
\text { y a utilizar los recursos disponibles. }\end{array}$ & No aplica \\
\hline
\end{tabular}

Nota: Solamente se tomaron en cuenta los diagnósticos que tenían que ver con las lesiones dérmicas de la paciente. 
1. Individualizado. Permite documentar los problemas del paciente, los objetivos del plan de cuidados y las acciones de enfermería para un paciente en concreto. Éste se tarda más tiempo en elaborar.

2. Estandarizado. Protocolo específico de cuidados, apropiado para aquellos pacientes que padecen los problemas normales o previsibles relacionados con el diagnóstico concreto o una enfermedad.

3. Estandarizado con modificaciones. Permite la individualización al dejar abiertas opciones en los problemas del paciente, los objetivos del plan de cuidados y las acciones de enfermería.

4. Computarizado. Requieren la captura previa en un sistema informático de los diferentes tipos de planes de cuidados estandarizados, son útiles si permiten la individualización de un paciente concreto.

El modelo de Virginia Henderson se incluye en la escuela de necesidades. Virginia afirma que el cuidado se centra en la independencia de la persona para satisfacción de sus necesidades fundamentales.

\section{Las 14 necesidades básicas de Virginia Henderson se} clasifican a continuación:

1. Respirar.

2. Comer y beber adecuadamente.

3. Eliminación de los desechos del organismo.

4. Moverse y mantener una postura adecuada.

5. Dormir y descansar.

6. Vestirse y desvestirse.

7. Mantener la temperatura corporal.

8. Mantener la higiene corporal.

9. Evitar los peligros del entorno.

10. Comunicarse.

11. Creencias y religión.

12. Trabajar.

13. Recreación y ocio.

14. Aprender.

Estas 14 necesidades humanas básicas son las que componen "los cuidados enfermeros", esferas en las que se desarrollan los cuidados. ${ }^{7}$ Los elementos más importantes de su teoría son: asistir a los pacientes en las actividades esenciales para mantener la salud, recuperarse de la enfermedad o alcanzar la muerte en paz, además introduce y/o desarrolla el criterio de independencia del paciente en la valoración de la salud.

\section{CARACTERÍSTICAS CLÍNICAS DEL PÉNFIGO VULGAR}

El pénfigo vulgar es una enfermedad autoinmune que afecta al tejido de la piel y mucosas, clínicamente se caracteriza por lesiones ampollosas que son frágiles de romper; la causa principal es la separación de los queratinocitos y la zona suprabasal de la epidermis, signo conocido como acantólisis. ${ }^{5}$ Caracterizado por la presencia de ampollas en la epidermis o las mucosas, evolucionan rápidamente hasta convertirse en erosiones superficiales. Afectan tanto las mucosas como la piel (todo lo que contenga formado de epitelio). Por lo tanto, también afectará a la cavidad bucal, la cual tarda en cicatrizar, es por eso que necesitan tratamiento y cuidados para prevenir infecciones. ${ }^{6}$ $\mathrm{Su}$ tratamiento farmacológico es a base de inmunosupresores como los glucocorticoides sistémicos; el fin es suprimir la formación de ampollas, habitualmente se emplean diferentes agentes inmunosupresores y antiinflamatorios coadyuvantes. Existen muchos tratamientos disponibles, sin embargo, se desconoce cuál es la opción de tratamiento más efectiva o más segura, o cuál es la mejor combinación. ${ }^{6}$

\section{PRESENTACIÓN DEL CASO CLÍNICO POR ENFERMERÍA}

Se trata de una mujer de 58 años de edad que tiene antecedente de diagnóstico médico de pénfigo vulgar. Inició su padecimiento en diciembre de 2012, y en enero de 2013 ingresó al Servicio de Terapia Intensiva, posteriormente en hospitalización, y siguiendo su trayectoria, fue dada de alta por mejoría clínica a su domicilio. Durante su estancia, fue paciente del personal de la Clínica de Heridas y Ostomías, donde se trataron sus lesiones dérmicas secundarias a las complicaciones del pénfigo: una úlcera por presión de IV grado. Actualmente es paciente externa de la Clínica de Heridas, es en este servicio donde se inicia la valoración para el presente caso.

\section{VALORACIÓN DE ENFERMERÍA}

La valoración especializada se realizó en lesiones dérmicas. El tratamiento de enfermería se basó en el cuidado que llevó a cabo con el proceso de cicatrización de las heridas a fin de que no hubieran complicaciones, abarcaran más tejido o se infectaran; asimismo, existió apoyo por parte de la Clínica del Dolor y la especialidad de Dermatología, estas disciplinas intervinieron para que el tratamiento fuera el adecuado para la paciente. Es una mujer que presenta lesiones dérmicas en antebrazos, cara posterior y espalda, con tejido de granulación, UPP IV en sacro con cavitación de aproximadamente $8 \mathrm{~cm}$ de ancho y con $5 \mathrm{~cm}$ de profundidad con tejido, de granulación exudado moderado, con lesiones dérmicas en los muslos en cara interna y externa con tejido de granulación.

Planes de cuidado: A continuación, se presentarán los diagnósticos de enfermería que corresponden a las necesidades alteradas de la valoración según el modelo. 
Cuadro II. Prioridad de atención de enfermería

1. Deterioro de la integridad tisular

2. Dolor agudo

3. Riesgo de infección

4. Disposición para mejorar el rol parenteral
A continuación se presentarán los diagnósticos por orden prioritario y por diagnóstico principal, ya que éstos conllevan al resto de los diagnósticos enfermeros (Cuadros I y II):

Cuadro III: Necesidad alterada: necesidad de evitar los peligros ambientales y los daños a otras personas

Indicios: UPP IV en sacro de $10 \mathrm{~cm}$ x $8 \mathrm{~cm}$ de dimensiones. Cavitada hacia las 11 horas de aproximadamente de $5 \mathrm{~cm}$. Heridas en miembros torácicos y espalda. Heridas en glúteos, heridas en muslos, en la cara interna y externo-posterior y heridas en piernas en cara externa.

\begin{tabular}{|c|c|c|}
\hline Código: 00004 & & \\
\hline $\begin{array}{l}\text { Diagnóstico: riesgo de infección r/c destrucción tisular, } \\
\text { aumento de la exposición ambiental a agentes patógenos }\end{array}$ & $\begin{array}{l}\text { Dominio: } 11 \\
\text { Seguridad y protección }\end{array}$ & $\begin{array}{l}\text { Clase: } 1 \\
\text { Infección }\end{array}$ \\
\hline
\end{tabular}

Resultados esperados NOC: 1842 control de la infección

\begin{tabular}{|c|c|c|c|c|c|c|}
\hline \multicolumn{2}{|c|}{ Indicadores } & \multicolumn{5}{|c|}{ Puntuación diana } \\
\hline 184202 & factores que contribuyen a la transmisión & 1 & 2 & 3 & 4 & 5 \\
\hline 184203 & prácticas que reducen la transmisión & 1 & 2 & 3 & 4 & 5 \\
\hline 184206 & procedimientos de control de la infección & 1 & 2 & 3 & 4 & 5 \\
\hline 184207 & importancia de la higiene de las manos & 1 & 2 & 3 & 4 & 5 \\
\hline 184209 & tratamiento de la infección diagnosticada & 1 & 2 & 3 & 4 & 5 \\
\hline \multicolumn{2}{|r|}{ Intervenciones NIC: control de infecciones } & \multicolumn{5}{|c|}{ DEPENDIENTE } \\
\hline Código & Intervenciones & \multicolumn{5}{|c|}{ Evaluación } \\
\hline 6540 & $\begin{array}{l}\text { - Cambiar el equipo de curación (en este caso fue cada tercer día). } \\
\text { - Mantener las técnicas de aislamiento. } \\
\text { - Intervención educativa a los familiares acerca del lavado de manos correcto } \\
\text { - Poner en práctica precauciones universales. } \\
\text { - Administrar terapia de antibacterianos. (El tratamiento incluyó un gel de plata, } \\
\text { antimicrobiano). }\end{array}$ & \multicolumn{5}{|c|}{$\begin{array}{l}\text { Fueron intervenciones funcionales para el control } \\
\text { del riesgo de la infección: } \\
\text { - Con técnica estéril } \\
\text { - Uso de gel antimicrobiano } \\
\text { - Lavado de manos } \\
\text { - La intervención educativa a los familiares } \\
\text { - Precauciones universales }\end{array}$} \\
\hline
\end{tabular}

Nota: Puntuación diana (aplicado a enfermería). 1: Ningún conocimiento; 2: Escaso conocimiento; 3: Conocimiento moderado; 4: Conocimiento sustancial; 5: Conocimiento extenso.

\section{Cuadro IV.}

Indicios: se observa el apoyo emocional de toda la familia y la disposición para ayudar a la paciente.

\section{Código: 00164}

Diagnóstico: Disposición para mejorar el rol parenteral

$\mathrm{m} / \mathrm{p}$. Manifiesta deseos de mejorar el rol parenteral y

es evidente la vinculación o unión. Las necesidades dependientes tratan de ser cubiertas por familiares.

\section{Dominio:

Rol/relaciones
Clase:1

Rol del cuidador

Resultados esperados NOC: 1600 conducta de adhesión

\begin{tabular}{|c|c|c|c|c|c|c|}
\hline \multirow{2}{*}{\multicolumn{2}{|c|}{$\frac{\text { Indicadores }}{160001 \text { pregunta cuestiones }}$}} & \multicolumn{5}{|c|}{ Puntuación diana } \\
\hline & & 1 & 2 & 3 & 4 & 5 \\
\hline \multirow{2}{*}{\multicolumn{2}{|c|}{$\begin{array}{l}160002 \text { busca información relacionada con la salud a partir de diversas fuentes } \\
160011 \text { realiza actividades de la vida diaria compatibles con su energía y tolerancia }\end{array}$}} & 1 & 2 & 3 & 4 & 5 \\
\hline & & 1 & 2 & 3 & 4 & 5 \\
\hline \multicolumn{2}{|r|}{ Intervenciones NIC: apoyo a la familia } & \multicolumn{5}{|c|}{ DEPENDIENTE } \\
\hline Código & Intervenciones & \multicolumn{5}{|l|}{ Evaluación } \\
\hline 7140 & $\begin{array}{l}\text { - Proporcionar seguridad a la familia acerca del cuidado que está recibiendo su paciente. } \\
\text { - Escuchar las inquietudes, sentimientos y preguntas de la familia. } \\
\text { - Favorecer una relación de confianza con la familia. } \\
\text { - Proporcionar ayuda para cubrir las necesidades del paciente. } \\
\text { - Facilitar oportunidades de visita a los familiares si procede. }\end{array}$ & \multicolumn{5}{|c|}{$\begin{array}{l}\text { Los familiares se sintieron en confianza; expresa- } \\
\text { ban que ellos observaban que su paciente estaba } \\
\text { recibiendo una mejor calidad de trato; se sentían } \\
\text { seguros y estaban en las mejores condiciones de } \\
\text { participación-cooperación. }\end{array}$} \\
\hline
\end{tabular}

Nota: Puntuación diana (aplicado al paciente). 1: Nunca demostrado; 2: Raramente demostrado; 3: A veces demostrado; 4: Frecuentemente demostrado; 5: Siempre demostrado 
Cuadro V. Necesidad alterada: higiene y protección de la piel

Indicios: UPP IV en sacro de $10 \mathrm{~cm} \times 8 \mathrm{~cm}$ de dimensiones. Cavitada hacia las 11 de aproximadamente $5 \mathrm{~cm}$. Con esfacelo y tejido necrótico.

\title{
Código: 00046
}

Diagnóstico: deterioro de la integridad tisular r/c.

factores mecánicos: presión m/p destrucción

Clase: 1

tisular

\author{
Seguridad y protección
}

Lesión física

Resultados esperados NOC: 1101

Indicadores

Puntuación diana

020401 úlceras por presión

Intervenciones NIC: cuidados de las úlceras por presión

DEPENDIENTE

\begin{tabular}{|c|c|c|}
\hline Código & Intervenciones & Evaluación \\
\hline 3520 & $\begin{array}{l}\text { - Controlar, la temperatura, edema, humedad y la apariencia de la piel circundante. } \\
\text { - Limpiar la piel alrededor de la úlcera suavemente con agua y jabón. } \\
\text { - Debridar la úlcera. } \\
\text { - Limpiar la úlcera con solución inyectable o estéril, con técnica aséptica. } \\
\text { - Anotar las características de la úlcera. } \\
\text { con curaciones cada tercer día }\end{array}$ & $\begin{array}{l}\text { Se notó un avance en la de reducción de dimensio- } \\
\text { nes de la UPP; se observó el tejido de granulación } \\
\text { sin datos de infección. Anteriormente con tejido } \\
\text { necrótico y esfacelo. Con el gel y el alginato de } \\
\text { calcio se logra desbridar el esfacelo y a través } \\
\text { de debridación mecánica se logra retirar tejido } \\
\text { necrótico.* }\end{array}$ \\
\hline
\end{tabular}

- Educación de la técnica de curación a familiares para realizarlo en casa cada tercer día.

*La evaluación se realizó 15 días después de la primera valoración.

Nota: Puntuación diana. (se aplica a úlcera por presión): 1: Grave; 2: Sustancial; 3: Moderado; 4: Leve 5: Ninguno

\section{Cuadro VI. Requisito alterado: prevención de peligros para la vida}

Indicios: heridas en miembros torácicos y espalda. Heridas en glúteos, heridas en muslos en cara interna y externo-posterior, heridas en piernas en cara externa.

\section{Código: 00007}

Diagnóstico: deterioro de la integridad tisular r/c enfermedad autoinmune $\mathrm{m} / \mathrm{p}$ destrucción de las capas de la piel

Dominio: 11 Seguridad y protección
Clase: 2

Lesión física

Resultados esperados NOC: 1101 integridad tisular: piel y membranas mucosas

\begin{tabular}{|c|c|c|c|c|c|}
\hline \multirow{2}{*}{$\begin{array}{l}\text { Indicadores } \\
110113 \text { integridad de la piel }\end{array}$} & \multicolumn{5}{|c|}{ Puntuación diana } \\
\hline & 1 & 2 & 3 & 4 & 5 \\
\hline 110116 Lesiones cutáneas & 1 & 2 & 3 & 4 & 5 \\
\hline \multicolumn{6}{|c|}{ DEPENDIENTE } \\
\hline
\end{tabular}

Código Intervenciones Evaluación

3660 1. Despegar los apósitos.

2. Monitorizar las características de la herida: color, olor, tejido y tamaño.

3. Medir el lecho de la herida.

4. Limpiar con solución estéril.

5. Aplicar hidrogel e hidrocoloide.

6. Ocluir con gasa y micropore.

7. Cambiar la gasa en caso de exceso de exudado de la herida.

8. Comparar y registrar regularmente cualquier cambio de la herida.

9. Promover educación a la familia y a la paciente para realizar la curación en casa y cuidados de la herida diaria. (quince días)

10. Documentar las características de la herida y el avance.
Las heridas iniciaron con infección, se brindó un tratamiento intensivo para controlarla, el tratamiento consistió en aplicación de gel de plata diariamente.

Quince días después la evolución de las heridas, se observa sin datos de infección, se encuentran las heridas con tejido de granulación.

Se prescribió el tratamiento que consistió con curaciones cada tercer día, aplicación de hidrogel e hidrocoloide; la paciente acude de forma ambulatoria a la clínica de heridas aproximadamente cada 20 días para curación y revaloración.

Nota: Puntuación diana (se aplica a las heridas).

1: Gravemente Comprometido; 2: Sustancialmente comprometido; 3: Moderadamente comprometido; 4: Levemente comprometido; 5: No comprometido 
Cuadro VII. Necesidad alterada: evitar los peligros ambientales y evitar lesionar a otras personas.

Indicios: presenta dolor al tacto. EVA (escala de visual análoga) de 6 puntos.

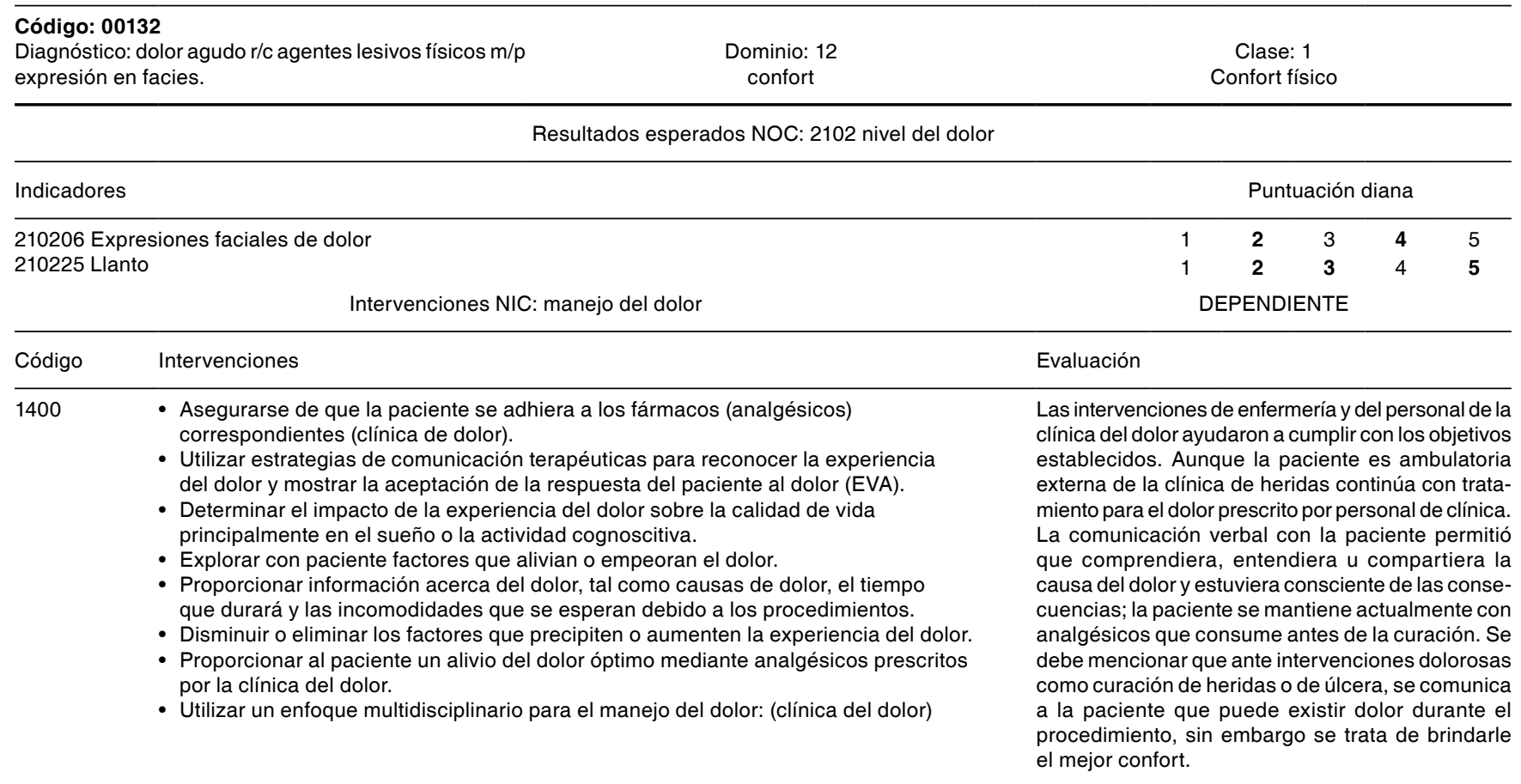

Nota: Puntuación diana. 1: Grave, 2: Sustancial; 3: Moderado; 4: Leve, 5: Ninguno.

Con los datos anteriores y los diagnósticos contemplados mostramos el Plan de Atención de Cuidados Enfermeros que se efectuaron en el presente caso (Cuadros III a VII):

\section{DISCUSIÓN}

La experiencia en llevar a cabo los planes de cuidados enfermeros específicos a esta persona, según el tratamiento enfermero, nos permitió la posibilidad de ejercer nuestra disciplina con un rigor metodológico en los procedimientos, intervenciones, seguimientos y valoraciones. Según las taxonomías empleadas por la NANDA, NIC, NOC, los resultados obtenidos con las intervenciones fueron favorables para la persona aquí tratada. La paciente hizo un continuo seguimiento al acudir a sus citas. Por otra parte, la información-comunicación por parte del equipo interdisciplinario fue de gran ayuda para que la familia se involucrara en el interés de fomentar el conocimiento y favorecer el bienestar de la paciente. La recuperación se llevó a cabo en un proceso de un año, la paciente y su familia observaron la evolución de sus lesiones, así como la efectividad del tratamiento-cuidado. Aunque esta enfermedad es de origen inmunológico, no quiere decir que desaparece, sin embargo, se puede controlar; es por este motivo que se le proporcionaron los elementos de aprendizaje necesario para su actuación cotidiana.

\section{BIBLIOGRAFÍA}

1. Aguilar-Serrano $L$ et al. El proceso enfermero y su nivel de autoeficacia. Rev Enferm Inst Mex Seguro Social. 2008; 16 (1): 3-6.

2. Alfaro Le-Fevre R. Aplicación del proceso enfermero fomentar el cuidado en colaboración. En: Perspectiva general del proceso enfermero. 5a edición, Barcelona: Masson; 2003: 5.

3. Blanco Fraile C, Saravía Lavín R, Lázaro Otero M. Metodología de la Enfermería [Internet]. Open Curse Ware Universidad de Cantabria; 2012. Disponible en: ocw-universi.ocw.universia.net/.../metodología-de-la-enfermería-

4. Griffith-Kenney J, Christensen PJ. Nursing Process: Application of theories, frameworks and models. 2nd, St Louis (MI): Mosby; 1986. Disponible en: http://www.ucol.mx/docencia/facultades/enfermeria/archivos1/ apuntes\%20utiles/PLACES.pdf. Consultado el 17 el febrero de 2014

5. Soares HD, Torres SC. Pênfigo vulgar: importância para o cirurgião dentista/Pemphigus vulgaris. Odontol USF. 1998; (10): 69-79.

6. Martin LK, Werth V, Villanueva E, Segall J, Murrell DF. Intervenciones para el pénfigo vulgar y el pénfigo foliáceo [revisión Cochrane traducida]. En: Biblioteca Cochrane Plus 2009. Oxford: Update Software Ltd. Disponible en: http: //www.update-software.com.

7. Wesley RL. Teorías y modelos de la enfermería. 2a edición. McGrawHill/Interamericano de México; 1997.

8. NANDA Internacional 2007-2008. Diagnósticos Enfermeros: Definición y Clasificación 2007-20068. Elsevier.

9. Clasificación de Resultados de Enfermería (NOC). Elsevier Mosby: Madrid; 2008.

10. Clasificación de Intervenciones de Enfermería (NIC). Elsevier Mosby: Madrid; 2008. 